\title{
Variação em propriedades da madeira de Pinus caribaea var. hondurensis e Pinus tecunumanii

\author{
Israel Luiz de Lima ${ }^{1^{*}}$ Ivanka Rosada de Oliveira $^{2}$ Juraci de Andrade Barbosa ${ }^{3}$ Maurício Ranzini ${ }^{1}$
}

${ }^{1}$ Instituto Florestal do Estado de São Paulo - IFEstação Experimental de Tupi, Estrada Rissieri Furlan, s/n - Tupi, CEP 13428-407 Piracicaba, SP, Brasil

${ }^{2}$ Universidade de São Paulo, Av. Pádua Dias, Piracicaba - SP, CEP 13418-900, Piracicaba, SP, Brasil

${ }^{3}$ Universidade Nove de Julho, R. Vergueiro, 235 CEP 01504-001, Liberdade, SP, Brasil

\begin{abstract}
Original Article
*Corresponding author: limailde@gmail.com

Keywords:

Pinus

Anatomy

Basic density

Tracheid

Palavras-chave:

Pinus

Anatomia

Densidade básica

Traqueíde

Received in

2020/04/27

RESUMO: Este estudo teve como objetivo caracterizar as propriedades da madeira de Pinus caribaea var. hondurensis e Pinus tecunumanii, em indivíduos de 50 anos de idade, na região de Itirapina, SP. Para isso, foram abatidas cinco árvores de cada espécie, e destas retirados um disco de $7 \mathrm{~cm}$ de espessura do diâmetro à altura do peito (DAP). De cada disco foram coletadas amostras em cinco posições diferentes, no sentindo medula-casca, para avaliar as seguintes propriedades: densidade básica, comprimento de traqueíde, espessura da parede de traqueíde, resistência ao cisalhamento e a retração volumétrica. Foi verificado que o Pinus caribaea var. hondurensis e o Pinus tecunumanii não diferem significativamente para as propriedades avaliadas. Entretanto, variaram significativamente, no sentido da medula-casca, para todas as propriedades. A densidade básica, comprimento de traqueíde e a retração volumétrica apresentaram uma tendência crescente no sentido da medula à casca. Para Pinus caribaea var. hondurensis ocorreram correlações positiva entre posição radial com: densidade básica, comprimento e espessura da parede de traqueídes. Com o Pinus tecunumanii ocorreram correlações entre posição radial com: densidade básica, espessura da parede de traqueíde e retração volumétrica. Em relação à utilização da madeira dessas espécies, ambas apresentam potencial de utilização para diversas finalidades tais como: serraria, fabricação de painéis e uso estrutural leve na construção civil.
\end{abstract}

Accepted on

2019/09/16

Published in

2021/01/12

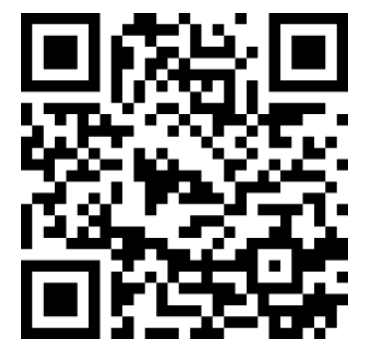

DOI: http://dx.doi.org/ 10.34062/afs.v7i4.10262

\section{(cc) BY}

\section{Variation in properties of the wood of Pinus caribaea var. hondurensis and Pinus tecunumanii}

ABSTRACT: This study aimed to evaluate if there are differences in wood properties between Pinus caribaea var. hondurensis and Pinus tecunumanii and their radial variations in individuals of 50 years of age, in the region of Itirapina, SP. For this, five trees of each species were felled. A disc of $7 \mathrm{~cm}$ thick in the region of the DBH was removed from each tree. Samples were collected in five different positions in the marrow-bark direction of each disc to determine the following properties of the wood: basic density, tracheid length, tracheid wall thickness, shear strength and shrinkage. It was found that Pinus caribaea var. hondurensis and Pinus tecunumanii do not differ significantly for the evaluated properties. However, they varied significantly, in the direction of pith to bark, for all properties. The basic density, tracheid length and shrinkage tend to increase in the direction of pith to bark of trunk of the trees. For Pinus caribaea var. hondurensis positive correlations between radial position with: basic density, tracheid length and tracheid wall thickness. For Pinus tecunumanii, there were correlations between radial position with: basic density, wall thickness of tracheid and shrinkage. Regarding the use of wood from these species, both have potential for different purposes such as: sawmill, panel manufacturing and light structural use in civil construction. 


\section{Introdução}

A madeira da Araucaria angustifolia foi muito utilizada no Brasil pelas indústrias madeireiras para fabricação de papéis, móveis, postes e construção civil. Porém, a exploração desordenada dessa espécie, desde o século passado reduziu drasticamente suas reservas (Cancian 2016). Devido a esse fato, entre 1960 e 1970, foram introduzidas no país espécies exóticas do gênero Pinus, de rápido crescimento, para aumentar os estoques de madeira e, assim, abastecer o setor madeireiro (Shimizu e Sebbenn 2008). Atualmente, os plantios de Pinus sp. ocupam 1,59 milhão de hectares e concentram-se no Paraná (42\%), Santa Catarina (34\%) e em São Paulo (8\%) (IBÁ 2019). Entre essas espécies que foram introduzidas, no Brasil, e que apresentam aptidão para produção de madeira, em escala comercial, podemos destacar o Pinus caribaea var. hondurensis e o Pinus tecunumanii.

Pinus tecunumanii tem grande potencial para plantios comerciais, dada a facilidade de adaptação as condições locais e a baixa incidência de foxtail (rabo de raposa), o ambiente ideal para o seu desenvolvimento é caracterizado por solos ácidos e argilo-arenosos, com profundidade de $40 \mathrm{~cm}$, boa drenagem e precipitação pluviométrica de $1.000 \mathrm{~mm}$ anuais (Shimizu e Sebbenn 2008). Essa espécie é oriunda do México, Guatemala, Belize, Honduras, El Salvador e Nicarágua, em geral cresce em regiões com altitude entre 600 a $2.600 \mathrm{~m}$ (Shimizu e Sebbenn 2008). No Brasil é cultivado no sudeste e centro-oeste, em regiões acima de $500 \mathrm{~m}$ de altitude, onde seu incremento médio anual é de $15 \mathrm{~m}^{3}$ por hectare (Lorenzi et al. 2018). O Pinus tecunumanii fornece madeira de cor amarelada de excelente qualidade para uso em construção civil, polpa para celulose, e, também, pode ser usado para paisagismo em geral (Lorenzi et al. 2018; Kronka et al. 2005; Shimizu e Sebbenn 2008). A madeira de Pinus tecunumanii normalmente apresenta variação interna em densidade, tanto no sentido medulacasca, quanto no sentido longitudinal do tronco, substancialmente menor do que outras espécies do gênero Pinus (Shimizu e Sebbenn 2008).

A espécie Pinus caribaea var. hondurensis é oriunda de Belize, Guatemala, Honduras e Nicarágua, em geral cresce e ocorre desde o nível do mar até $850 \mathrm{~m}$ de altitude. No Brasil é uma das espécies mais plantadas em decorrência da facilidade de obtenção de sementes. Essa espécie produz madeira de cor branca ou amarelada, pode ser utilizada para resinagem, construção civil e paisagismo em geral. Sua madeira é de densidade moderada a baixa, mas de grande utilidade geral (Lorenzi et al. 2018; Kronka et al. 2005).

Para Santos et al. (2018) diversas espécies do gênero Pinus estão sendo utilizados no setor madeireiro brasileiro, das quais, o Pinus caribaea var. hondurensis é uma que se destaca para a produção de madeira e resina. Com o objetivo de ofertar genótipos do gênero Pinus para o mercado visando à exploração de madeira e resina, a avaliação e seleção de material mais produtivo tornam-se ainda necessárias.

De maneira geral temos boas informações a respeito do crescimento, adaptação, e uso da madeira do Pinus caribaea var. hondurensis e Pinus tecunumanii, no Brasil, porém, ainda são imprescindíveis mais estudos principalmente referentes à qualidade da madeira, com destaque para as propriedades físicas, mecânicas e dimensões anatômicas, que dão subsídio na determinação do potencial de utilização comercial. Para a produção de madeira mais competitivas, deve-se visar à obtenção de melhor qualidade, o que implica a incorporação de novos parâmetros de avaliação de aptidão da madeira para uso sólido.

As características anatômicas estão intimamente relacionadas com as propriedades da madeira e, portanto, com seu processamento tecnológico. Por esta razão, o comprimento e a espessura da parede celular de traqueíde são considerados de grande importância na avaliação da qualidade da madeira de espécies de Pinus (Winck, et al. 2015). Outra propriedade importante é a densidade que controla a amplitude das mudanças dimensionais que ocorrem com a madeira, em função das mudanças no conteúdo de umidade abaixo do ponto de saturação das fibras (Latorraca et al. 2000).

Outras das questões fundamentais para definir a qualidade da madeira para a utilização comercial é saber qual é a proporção de madeira juvenil existente em uma tora e como se comporta a sua variação no sentido medula-casca. Essa é uma característica que depende fundamentalmente da idade da árvore, bem como do ambiente e do manejo (Malan 1995). Entretanto, em uma mesma idade e em condições semelhantes de ambiente e de manejo, pode-se verificar que existe grande variabilidade nessa proporção (Hillis e Brown 1984).

Sendo assim, o objetivo desse trabalho foi avaliar as diferenças nas propriedades da madeira das árvores de Pinus caribaea var. hondurensis e Pinus tecunumanii, aos 50 anos de idade e verificar se existe variação no sentido medula-casca.

\section{Material e Métodos}

Foram utilizadas amostras de madeiras obtidas de árvores de plantio experimental de Pinus caribaea var. hondurensis e Pinus tecunumanii, da região de Itirapina SP, com idades de 50 anos. A área em estudo situa-se entre as coordenadas $22^{\circ} 00^{\prime} \mathrm{S}$ e $22^{\circ} 15^{\prime} \mathrm{S}$ de latitude e $47^{\circ} 45^{\prime} \mathrm{W}$ e $48^{\circ} 00^{\prime} \mathrm{W}$ de longitude. Os solos são areia quartzosa profunda e hidromórfico. O clima da região é tropical com inverno seco, com temperaturas variando de $16,4^{\circ} \mathrm{C}$ (estação seca - abril a novembro) a $24,8^{\circ} \mathrm{C}$ (estação chuvosa - dezembro a março) (Zanchetta et al. 2006). 
A região possui altitude média de $801 \mathrm{~m} \mathrm{e}$ precipitação anual média de $1.377 \mathrm{~mm}$ (Flores et. al., 2016).

As amostras foram coletadas de cinco árvores (repetições) de cada espécie selecionadas aleatoriamente, obedecendo a média do diâmetro à altura do peito $\mathrm{DAP}(1,30 \mathrm{~m}$ do solo $)$ de acordo com os dados obtidos no inventário florestal. De cada árvore selecionada, foi retirado um disco de $7 \mathrm{~cm}$ de espessura, na altura de DAP (Tabela 01). Em cada um dos discos foi retirado um bloco amostral representativo da região da medula até casca. Do bloco amostral foram retiradas cinco amostras de 5 $\mathrm{cm} \times 2,5 \mathrm{~cm} \times 5 \mathrm{~cm}$, representativa das posições 0 , $25,50,75$ e $100 \%$ do raio da árvore para estudo da variação medula-casca.

As propriedades e dimensões anatômicas da madeira avaliadas foram: densidade básica (DB), comprimento de traqueíde (CT), espessura da parede de traqueíde $(\mathrm{EPT})$, cisalhamento $\left(\mathrm{f}_{\mathrm{v} 0}\right)$ e retração volumétrica $\left(\varepsilon_{\mathrm{v}}\right)$.

Tabela 1. Valores médios dos diâmetros à altura do peito (DAP) e alturas totais (HT) das árvores amostradas nas populações de Pinus caribaea var. hondurensis e Pinus tecunumanii, de 50 anos de idade.

\begin{tabular}{ccc|ccc}
\hline \multicolumn{3}{c}{ Pinus tecunumanii } & \multicolumn{3}{c}{ Pinus caribaea var. hondurensis } \\
\hline Árvore & $\mathrm{DAP}(\mathrm{cm})$ & $\mathrm{HT}(\mathrm{m})$ & Árvore & $\mathrm{DAP}(\mathrm{cm})$ & $\mathrm{HT}(\mathrm{m})$ \\
\hline 1 & 27,76 & 27,00 & 1 & 31,69 & 32,00 \\
2 & 30,30 & 30,00 & 2 & 21,79 & 22,00 \\
3 & 33,30 & 33,00 & 3 & 18,63 & 19,00 \\
4 & 21,82 & 22,00 & 4 & 20,38 & 21,00 \\
5 & 22,15 & 23,00 & 5 & 26,35 & 27,00 \\
\hline Média & 27,07 & 27,00 & Média & 23,77 & 24,20 \\
\hline
\end{tabular}

\section{Densidade Básica (DB)}

Para a obtenção da densidade básica foi utilizado o método da balança hidrostática conforme NBR 11941 (ABNT 2003). Os corpos de prova de $2,0 \mathrm{~cm} \times 2,0 \mathrm{~cm}$ de lado e comprimento ao longo das fibras de 3,0 cm, retirados de cada amostra, foram saturados por um período de aproximadamente um mês, onde se pode obter a massa totalmente saturada e imersa de cada amostra. Posteriormente, foram secos em estufa até atingirem a massa seca constante a $103 \pm 2$ oC. Foi utilizada a expressão (1):

$$
D B=\frac{P_{s}}{P_{u}-P_{i}}
$$

Em que: $\mathrm{DB}=$ densidade básica $(\mathrm{g} / \mathrm{cm} 3) ; \mathrm{Ps}=$ massa seca $(\mathrm{g}) ; \mathrm{Pu}=$ massa saturada $(\mathrm{g})$, e $\mathrm{Pi}=$ massa do recipiente com água $(\mathrm{g})$.

\section{Resistência ao cisalhamento tangencial aos anéis de crescimento $(f v 0)$}

Para este ensaio foram utilizados corpos de prova com dimensões nominais de $2 \times 2 \times 3 \mathrm{~cm}$, com $5 \mathrm{~cm} 2$ de área de cisalhamento ou seja $(2 \mathrm{~cm} \times 2,5$ $\mathrm{cm})$. Um lado da área cisalhada foi de $2 \mathrm{~cm}$, imposta pela dimensão da seção do disco velocidade de aplicação de carga foi de 2,5 $\mathrm{MPa} / \mathrm{min}$ (N/mm2/min), especificadas na norma NBR 7190 (ABNT 1997).

Retração volumétrica (cv)
A retração volumétrica foi obtida de corpos de prova de 2,0 cm x 2,0 cm de lado e comprimento ao longo das fibras de $3,0 \mathrm{~cm}$. Os corpos de prova foram saturados por um período de aproximadamente um mês, possibilitando obter os seus volumes completamente saturados. Posteriormente, foram secos em estufa até atingirem a massa seca constante a $1032 \mathrm{oC}$, o que resultou na obtenção do volume seco de cada corpo de prova MB 26 (ABNT 1940) e na determinação desta propriedade foi utilizada a expressão (2):

$$
\varepsilon_{\mathrm{v}}=\frac{V_{\text {sat }}-V_{\mathrm{sec} a}}{V_{\text {sat }}} 100
$$

Em que: $\varepsilon v=$ retração volumétrica, \%; Vsat= volume da madeira saturada, $\mathrm{cm} 3$, e Vseca $=$ volume da madeira seca, cm3.

\section{Dimensões dos traqueídes}

De cada disco, foram retiradas amostras para medição das dimensões dos traqueídes da madeira. As células foram dissociadas pelo processo de maceração (Johansen 1940). Para essa fase, fragmentos do lenho foram transferidos para frascos de vidro e, em seguida, embebidos com solução macerante de ácido acético glacial e água oxigenada 120 vol. (1:1), conduzidos à estufa $60^{\circ} \mathrm{C}$, por $48 \mathrm{~h}$; após escoada, foram lavados com água. Para avaliação das dimensões, as células dissociadas do 
lenho foram coradas com safranina e montadas em lâminas semipermanentes com glicerina $30 \%$.

As dimensões dos traqueídes da madeira das espécies de Pinus foram medidas com auxílio de microscópio trinocular, acoplado à câmera de vídeo em sistema de análise de imagens. Foi efetuado o processo de medição com auxílio do software Image Pro-Plus, das seguintes dimensões: Comprimento do traqueíde $(\mu \mathrm{m})$ e Espessura da parede do traqueíde. Para calcular a espessura da parede dos traqueídes, utilizou-se da expressão (3):

$$
E P T=\frac{D-d}{2}
$$

Em que: $\mathrm{EPT}=$ espessura da parede do traqueíde $(\mu \mathrm{m}) ; \mathrm{D}=$ diâmetro do traqueíde $(\mu \mathrm{m}), \mathrm{e} \mathrm{d}=$ diâmetro do lume do traqueíde $(\mu \mathrm{m})$.

\section{Análise estatística}

$\mathrm{Na}$ avaliação do experimento foi aplicado inicialmente o teste de homogeneidade de variância e, para isso, se utilizou o teste de Hartley. Posteriormente, se efetuou a análise de variância e aplicou-se o teste de Tukey para a comparação das médias. O teste de Tukey foi utilizado para a determinação das médias que diferiram entre si, sempre que foi observada diferença significativa ao nível de $5 \%$ de probabilidade dos tratamentos no teste $\mathrm{F}$.

Foi realizado um estudo de relações entre as propriedades avaliadas utilizando-se a análise de regressão linear simples, ao nível de significância de $95 \%$.

Os resultados obtidos das propriedades foram analisados estatisticamente com o auxílio do procedimento estatístico PROC GLM e PROC REG do programa estatístico SAS (SAS 1999).

\section{Resultados e discussões}

A Tabela 2 mostra a análise de variância efetuada para densidade básica, comprimento de traqueíde, espessura da parede de traqueíde, cisalhamento e retração volumétrica de Pinus tecunumanii e Pinus caribaea var. hondurensis. De acordo com os resultados observados verifica-se que não ocorreram diferenças significativas, ao nível de $5 \%$ de probabilidade, entre as espécies para nenhuma das variáveis. No entanto, foram constatadas diferenças entre as posições radiais, no tronco da árvore para todas as propriedades. Também, foi observado que não foram significativos os efeitos da interação espécie $\mathrm{x}$ posição radial para todas as variáveis. Então não existe uma dependência entre esses fatores, o que demonstra que o padrão de variação das variáveis é o mesmo para todos os tratamentos (Tabela 2).

Tabela 2. Resumo da análise da variância para densidade básica (DB), comprimento de traqueíde (CT), espessura da parede de traqueíde (EPT), cisalhamento $\left(\mathrm{f}_{\mathrm{v} 0}\right)$ e retração volumétrica $\left(\varepsilon_{\mathrm{v}}\right)$ da madeira Pinus caribaea var. hondurensis e Pinus tecunumanii, de 50 anos de idade.

\begin{tabular}{lcccccc}
\hline Causa de variação & GL & \multicolumn{5}{c}{ Quadrado médio } \\
\cline { 3 - 6 } & & DB & CT & EPT & $\mathrm{f}_{\mathrm{v} 0}$ & $\varepsilon_{\mathrm{v}}$ \\
& & $\left({\left.\mathrm{g} . \mathrm{cm}^{-3}\right)}\right.$ & $(\mathrm{mm})$ & $(\mu \mathrm{m})$ & $(\mathrm{MPa})$ & $(\%)$ \\
\hline Espécie (E) & 1 & $0,0019^{\text {n.s. }}$ & $0,0031^{\text {n.s. }}$ & $0,56^{\text {n.s. }}$ & $0,0900^{\text {n.s. }}$ & $9,5492^{\text {n.s. }}$ \\
Posição Radial (PR) & 4 & $0,0148^{*}$ & $0,7947^{*}$ & $7,85^{*}$ & $4,1367^{*}$ & $42,97^{*}$ \\
$($ E) x (PR) & 4 & $0,0027^{\text {n.s. }}$ & $0,1721^{\text {n.s. }}$ & $0,49^{\text {n.s. }}$ & $0,0804^{\text {n.s. }}$ & $10,3454^{\text {n.s. }}$ \\
\hline Média & & 0,45 & 3,76 & 8,04 & 10,42 & 11,74 \\
\hline CV $_{\mathrm{e}}(\%)$ & & 10,88 & 9,12 & 18,75 & 9,86 & 28,07
\end{tabular}

n.s.: não significativo *: significativo ao nível de $5 \%$ de significância e $\mathrm{CV}_{\mathrm{e}}$ : coeficiente de variação experimental

De acordo com os valores médios apresentados para densidade básica de Pinus tecunumanii e Pinus caribaea var. hondurensis, não há diferença significativa para essa propriedade (Tabela 2). Pode-se considerar que essas espécies produzem madeira leve, pois apresentaram valores inferiores a $0,50 \mathrm{~g} . \mathrm{cm}^{-3}$ (Zenid 2007). Moura et al. (2004) verificaram valores de densidade básica de 0,42 e $0,46 \mathrm{~g} . \mathrm{cm}^{-3}$ para Pinus tecunumanii com 12 e 17 anos, respectivamente e Trianoski et al. (2013) observaram valores de 0,49 g.cm ${ }^{-3}$, em árvores de 18 anos de idade. Oluwafemi (2007) constatou valores de 0,49 g.cm ${ }^{-3}$ para densidade básica de Pinus caribaea com idade de 25 anos. Trianoski et al. (2013), reportou valor de 0,42 g.cm ${ }^{-3}$ para o Pinus caribaea var. bahamensis de 18 anos de idade. Para Melo (2015), a idade e seus fatores de interação influenciaram significativamente os valores de densidade da madeira de Pinus taeda, ou seja, neste caso a densidade de madeira aumentou com a idade. 
Para o comprimento e a espessura da parede de traqueíde, as espécies apresentaram valores semelhantes estatisticamente (Tabela 3). Valor de 4,23 mm para comprimento de traqueíde de Pinus caribaea com idade de 25 anos foi constatado por Oluwafemi (2007). Correa e Bellote (2011) verificaram valores de $10,5 \mu \mathrm{m}$ para a espessura da parede de traqueíde de Pinus caribaea var. hondurensis com 11 anos de idade. Shimoyama e Wiecheteck (1993) obtiveram valores de $10 \mu \mathrm{m}$ para Pinus tecunumanii com 12 anos de idade, Torres et. al. (2005) constataram para essa mesma espécie também com 12 anos valores de 9,48 $\mu \mathrm{m}$.

Tabela 3. Médias para densidade básica (DB), comprimento de traqueíde (CT), espessura da parede de traqueíde (EPT), cisalhamento $\left(\mathrm{f}_{\mathrm{v} 0}\right)$ e retração volumétrica $\left(\varepsilon_{\mathrm{v}}\right)$ da madeira Pinus caribaea var. hondurensis e Pinus tecunumanii, de 50 anos de idade.

\begin{tabular}{cccccc} 
Tratamento & $\begin{array}{c}\mathrm{DB} \\
\left(\mathrm{g} . \mathrm{cm}^{-3}\right)\end{array}$ & $\begin{array}{c}\mathrm{CT} \\
(\mathrm{mm})\end{array}$ & $\begin{array}{c}\mathrm{EPT} \\
(\mu \mathrm{m})\end{array}$ & $\begin{array}{c}\mathrm{f}_{\mathrm{v} 0} \\
(\mathrm{MPa})\end{array}$ & $\begin{array}{c}\varepsilon_{\mathrm{v}} \\
(\%)\end{array}$ \\
\hline Pinus tecunumanii & $0,46^{\mathrm{A}}$ & $3,77^{\mathrm{A}}$ & $7,94^{\mathrm{A}}$ & $10,46^{\mathrm{A}}$ & $11,31^{\mathrm{A}}$ \\
Pinus caribaea var. hondurensis & $0,44^{\mathrm{A}}$ & $3,75^{\mathrm{A}}$ & $8,93^{\mathrm{A}}$ & $10,38^{\mathrm{A}}$ & $12,18^{\mathrm{A}}$ \\
\hline Posição radial (0\%) & $0,40^{\mathrm{b}}$ & $3,38^{\mathrm{c}}$ & $6,97^{\mathrm{b}}$ & $9,97^{\mathrm{b}}$ & $9,01^{\mathrm{b}}$ \\
Posição radial (25\%) & $0,44^{\mathrm{ab}}$ & $3,56^{\mathrm{bc}}$ & $7,82^{\mathrm{ab}}$ & $10,09^{\mathrm{ab}}$ & $10,14^{\mathrm{ab}}$ \\
Posição radial (50\%) & $0,45^{\mathrm{ab}}$ & $3,86^{\mathrm{ab}}$ & $8,07^{\mathrm{ab}}$ & $9,89^{\mathrm{b}}$ & $12,51^{\mathrm{ab}}$ \\
Posição radial (75\%) & $0,47^{\mathrm{a}}$ & $4,05^{\mathrm{a}}$ & $8,87^{\mathrm{a}}$ & $10,79^{\mathrm{ab}}$ & $13,02^{\mathrm{ab}}$ \\
Posição radial $(100 \%)$ & $0,50^{\mathrm{a}}$ & $3,95^{\mathrm{ab}}$ & $8,77^{\mathrm{a}}$ & $11,39^{\mathrm{a}}$ & $14,02^{\mathrm{a}}$ \\
\hline
\end{tabular}

Obs: Médias seguidas de letras diferentes em uma mesma coluna diferem entre si (ao nível de 5\% de significância)

Em relação à resistência ao cisalhamento, as espécies também apresentaram valores estatisticamente iguais (Tabela 3). Trianoski et al. (2014) comprovaram para Pinus caribaea var. hondurensis valor de 10,49 MPa e 11,42 MPa para Pinus tecunumanii, ambas espécies com 18 anos de idade.

Verificou-se, que de maneira geral, não houve variação significativa para as propriedades e dimensões anatômicas, entre as duas espécies em estudo. Entretanto, de acordo com literatura consultada era de se esperar que o Pinus tecunumanii apresentasse valores maiores de densidade e resistência mecânica, em relação ao Pinus caribaea, ao ponto de se diferenciarem significativamente entre si, porém isso não ocorreu. Uma das possíveis explicação desses resultados pode ser o fato de fatores ambientais e genéticos terem sido mais influentes na determinação das propriedades da madeira. Outra hipótese, para isto, seria em relação a idade de 50 anos, dessas árvores que nestas condições, podem ter se tornadas menos resistentes, ou seja, esse efeito da resistência com a idade pode ter sido diluído com, o passar do tempo, e assim contribuiu para que essas espécies não se diferenciassem significativamente.

A partir da análise da Tabela 3, observa-se as variações das propriedades em função da posição radial. Pode-se relatar que ocorreram tendências de crescimento dos valores de quase todas variáveis, no sentido da medula até a casca, onde os menores valores estão próximos da medula, e os maiores, nas proximidades da casca. De maneira geral, de acordo com a literatura espécies de Pinus apresentam um aumento na densidade básica no sentido medulacasca (Ballarin e Palma 2003; Souza et al. 2007).

Para explicar melhor essa tendência foi realizada a análise de correlação entre posição radial $(\mathrm{em}, \mathrm{cm})$ e as variáveis DB, CT, EPT, f fv e $\varepsilon_{\mathrm{v}}$ em separado por espécie (Tabela 4). De acordo com os resultados obtidos, verifica-se que para o Pinus caribaea a densidade básica, comprimento e espessura de traqueíde apresentaram correlações significativas com a distância do raio da tora da árvore. Para o Pinus tecunumanii a densidade básica, espessura de traqueíde e a retração volumétrica apresentaram correlações significativas (Tabela 4).

Para as variáveis que tiveram as melhores correlações significativas, foi feita a análise de regressão para obter as equações que podem explicar melhor essa relação entre elas (Figuras 1 e 2).

Para o Pinus tecunumanii, comparando-se os resultados obtidos da densidade básica, retração volumétrica e a espessura da parede traqueíde, podese verificar que ocorreu aumento no sentido medulacasca (Figuras 1a, 1b e 1c); isso pode ser explicado pelo aumento proporcional de madeira adulta em relação à madeira juvenil. Ballarin e Palma (2003) e Souza et al. (2007) para Pinus taeda também verificaram que a densidade da madeira e as dimensões dos traqueídes aumentam no sentido da medula-casca. Rios et al. (2018) também constataram que ocorreu aumento da densidade básica da parte interna para a parte externa, no sentido radial da madeira para Pinus patula. Essa mesma tendência foi observada por Melo et al. 
(2013) para Pinus elliottii, onde os fatores posição axial e posição radial influenciaram significativamente os valores de densidade, sendo que a densidade da madeira reduziu da base para o topo e aumentou no sentido medula-casca. Segundo
Palermo et al. (2013), a idade de transição do lenho juvenil para adulto de Pinus elliottii se comporta diferentemente entre as variáveis: comprimento de traqueídes e densidade da madeira.

Tabela 4. Coeficientes de correlação de Pearson entre raio, densidade básica (DB), comprimento de traqueíde $(\mathrm{CT})$, espessura da parede de traqueíde $(\mathrm{EPT})$, cisalhamento $\left(\mathrm{f}_{\mathrm{v} 0}\right)$ e retração volumétrica $\left(\varepsilon_{\mathrm{v}}\right)$ da madeira de Pinus caribaea var. hondurensis e Pinus tecunumanii de 50 anos.

\begin{tabular}{cccc}
\hline \multicolumn{2}{c|}{ Pinus caribaea var. hondurensis } & \multicolumn{2}{|c}{ Pinus tecunumanii } \\
\hline Raio $(\mathrm{cm})$ & $0,92^{* *}$ & DB & $0,91^{*}$ \\
DB & $0,88^{*}$ & $\mathrm{CT}$ & $0,85^{\text {n.s. }}$ \\
EPT & $0,92^{*}$ & EPT & $0,89^{*}$ \\
$\mathrm{f}_{\mathrm{v} 0}$ & $0,83^{\text {n.s. }}$ & $\mathrm{f}_{\mathrm{v} 0}$ & $0,88^{\text {n.s. }}$ \\
$\varepsilon_{\mathrm{v}}$ & $0,75^{\text {n.s. }}$ & $\varepsilon_{\mathrm{v}}$ & $0,98^{* *}$ \\
\hline
\end{tabular}

Onde: ** significativo ao nível de $1 \%$ de significância; * significativo ao nível de $5 \%$ de significância n. $\mathrm{s} .=$ não significativo.

Gonçalez et al. (2018) relatam que para o Pinus caribaea var. hondurensis a densidade básica e retração volumétrica aumentam no sentido medula casca, sendo que isto ocorreu devido à diminuição da espessura dos anéis de crescimento no sentido medula-casca.

Essas relações positivas entre posição radial e a densidade básica, comprimento de traqueíde, retração volumétrica, também foram constatadas para Pinus caribaea var. hondurensis (Figuras 2a, 2b e 2c). O modelo de regressão linear foi o que melhor representou as relações entre essas propriedades e a posição radial (Figuras 1 e 2). Como pode ser observado, temos uma forte dependência entre a posição radial e essas propriedades estudadas para ambas as espécies (Figuras 1 e 2). De acordo com as equações obtidas, pode-se estimar essas propriedades em função da posição radial. Porém, temos que considerar que: diferença muito acentuada em propriedades da madeira, no sentido radial, pode ser prejudicial para alguns usos industriais que requerem madeira mais homogênea possível como por exemplo o uso estrutural da madeira.

De acordo com os resultados das propriedades das madeiras apresentados por ambas as espécies, com idade de 50 anos, confirma-se o potencial de utilização para diversos usos tais como por exemplo: fabricação de painéis, uso estrutural leve na construção civil e serraria, sendo então uma alternativa para suprir a demanda de matéria prima do setor industrial.

\section{Conclusões}

De acordo com os resultados apresentados, pode-se concluir que:

A densidade básica, comprimento de traqueíde, espessura da parede de traqueíde, resistência ao cisalhamento e retração volumétrica não diferem significativamente entre as espécies Pinus tecunumanii e Pinus caribaea var. hondurensis.

A densidade básica, resistência ao cisalhamento, retração volumétrica, comprimento de traqueíde e a espessura da parede de traqueíde apresentam variações no sentido da medula para a casca dos troncos das árvores.

Para Pinus caribaea var. hondurensis ocorre correlações positivas entre posição radial com densidade básica, comprimento de traqueíde e espessura da parede de traqueíde.

Em relação ao Pinus tecunumanii ocorre as seguintes correlações positivas: posição radial com densidade básica, espessura da parede de traqueíde e retração volumétrica.

No caso do Pinus caribaea var. hondurensis a densidade básica, comprimento de traqueíde e espessura da parede de traqueíde apresentam uma tendência de aumentar no sentido da medula para casca.

Já para o Pinus tecunumanii a densidade básica, espessura da parede de traqueíde e retração volumétrica apresentam uma tendência de aumentar no sentido da medula para casca.

Ambas espécies confirmaram o potencial de utilização para diversas finalidades tais como: serraria, fabricação de painéis e uso estrutural leve na construção civil. 
Lima et al.

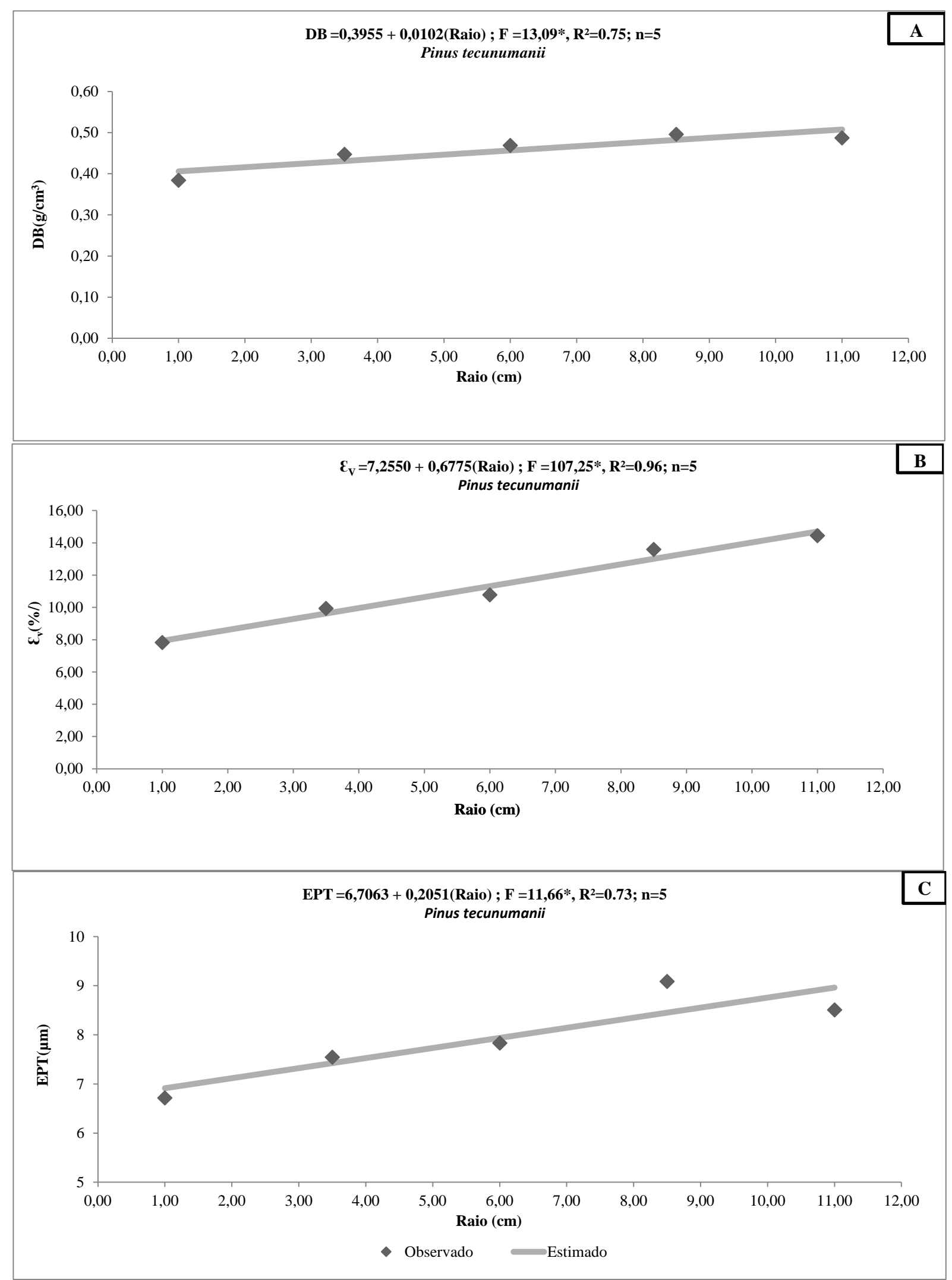

Figura 1. Relações entre posição radial (PR) e densidade básica (DB) [a], retração volumétrica $\left(\varepsilon_{v}\right)[b]$ e espessura da parede de traqueíde (EPT) [c] para Pinus tecunumanii de 50 anos de idade. 
Lima et al.

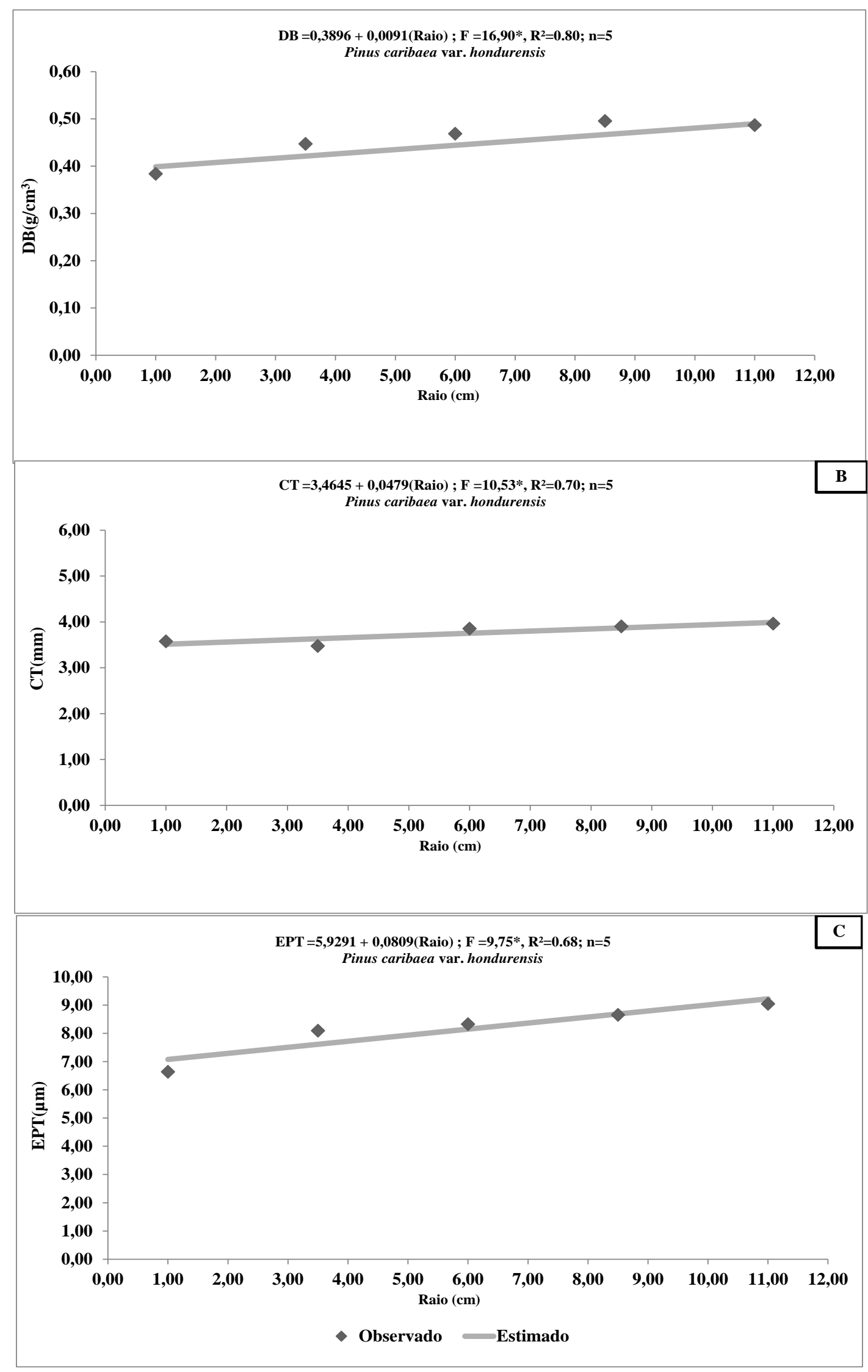

Figura 2. Relações entre posição radial (Raio) e densidade básica (DB) [a], comprimento de traqueíde (CT) [b] e retração volumétrica $\left(\varepsilon_{\mathrm{v}}\right)$ e para Pinus caribaea var. hondurensis de 50 anos de idade 


\section{Referencias bibliográficas}

ABNT. Associação Brasileira de Normas Técnicas (1940). MB 26/40: Ensaios Físicos e Mecânicos de madeira. Rio de Janeiro. $16 \mathrm{p}$.

ABNT. Associação Brasileira de Normas Técnicas (2003). NBR 11941: Madeira: determinação da densidade básica. Rio de Janeiro. 6 p.

ABNT. Associação Brasileira de Normas Técnicas (1997). NBR 7190: Projeto de Estruturas de Madeiras. São Paulo. 107 p.

Ballarini AW, Palma HAL (2003). Propriedades de resistência de madeira juvenil e adulta de Pinus taeda L. Revista Árvore, 27(3):371-380. doi.org/10.1590/S0100-67622003000300014

Cancian PRM (2016). Araucária: raízes da industrialização. Caxias do Sul: Educs. 173 p.

Corrêa RS, Bellote AFJ (2011). Influência do solo na dimensão dos traqueídeos e densidade da madeira em Pinus caribaea var. hondurensis. Pesquisa Florestal Brasileira, 31(66):93-102. dx.doi.org/10.4336/2011.pfb.31.66.93.

Goncalez JC, Santos N, Silva Junior FG, Souza RS, De Paula MH (2018). Growth ring width of Pinus caribaea var. hondurensis and its relationship with wood proprieties. Scientia Forestalis, 46(118):309317. dx.doi.org/10.18671/scifor.v46n118.16.

Flores TB, Alvares CA, Souza VC, Stape JL. (2016). Eucalyptus no Brasil: Zoneamento climático e guia para identificação. Piracicaba: IPEF. 448 p.

Hillis WH, Brown AG (1984). Eucaliptos for wood production. Melbourne: CSIRO. 434 p.

IBÁ. Indústria Brasileira de Árvores (2019).

Relatório Ibá 2019. São Paulo: Ibá. 80 p.

Disponível

em:<https://iba.org/datafiles/publicacoes/relatorios/ iba-relatorioanual2019.pdf $>$, Acesso em: $19 \mathrm{dez}$. 2019 .

Johansen DA (1940). Plant microtecniques. New York: McGraw-Hill. 523 p.

Kronka FJN, Bertolani F, Ponce RH (2005). A cultura de Pinus no Brasil. São Paulo: Páginas \& letras. Sociedade Brasileira de Silvicultura. p. 154.

Latorraca JVF, Albuquerque CEC (2000). Efeito do rápido crescimento sobre as propriedades da madeira. Floresta e Ambiente, 7(1):279-291.
Lorenzi H, Bacher LB, Torres MAV (2018). Árvores e Arvoretas exóticas no Brasil: madeireiras, ornamentais e aromáticas. Nova Odessa: Instituto Plantarum. 464 p.

Malan FS (1995). Eucalipts improvement for lumber production. In: I Seminário Internacional $e$ Utilização da Madeira de Eucalipto para Serraria, Piracicaba, Brasil.

Melo RR (2015). Radial and longitudinal variation of Pinus taeda L. wood basic density in different ages. Revista de Ciências Agrárias, 58(2):192-197. doi.editoracubo.com.br/10.4322/rca.1839.

Melo RR, Silvestre R, Oliveira TM, Pedrosa TD (2013).Variação radial e longitudinal da densidade básica da madeira de Pinus elliottii Engelm. com diferentes idades. Revista Ciência da Madeira, 4(1):83-92. dx.doi.org/10.15210/cmad.v4i1.4057.

Moura VPG, Vale AT, Isaias FB (2004). Comparação entre dois métodos de avaliação da variabilidade genética em volume, densidade básica da madeira e matéria seca de Pinus tecunumanii (Schwd) Eguiluz e Perry. Ciência Florestal, 14(1):77-84. dx.doi.org/10.5902/198050981783.

Oluwafemi OA (2007). Wood properties and selection for rotation length in Caribbean pine (Pinus caribaea Morelet) grown in Afaka, Nigeria. American-Eurasian Journal of Agricultural \& Environmental Sciences. 2(4):359-363. idosi.org/aejaes/jaes2(4)/6.pdf

Palermo GPDM, Latorraca JVF, Severo ETD, Do Nascimento AM, Rezende, MA (2013). Delimitação entre os lenhos juvenil e adulto de Pinus elliottii Engelm. Revista Árvore, 37(1): 91-200. doi.org/10.1590/S0100-67622013000100020.

Rios PDA, Vieira HC, Pereira GF, Turmina E, Nicoletti MF (2018). Variação radial e longitudinal da densidade básica da madeira de Pinus patula. Pesquisa Florestal Brasileira, 38(1):1-5. dx.doi.org/10.4336/2018.pfb.38e201501016.

Santos W, Silva MSC, Deniz LD, Kieras WS, Shimizu JY, Sousa VA, Aguiar AV (2018). Identificação de Procedências e Progênies de Pinus maximinoi com potencial produtivo para madeira. Scientia Forestalis, 46(117):127-136. dx.doi.org/10.18671/scifor.v46n117.12.

SAS. Statistical Analysis System (1999). SAS Procedures guide: version 8 (TSMO). Cary: SAS.

Sebbenn AM, Shimizu JY, Aguiar AV (2008). Produção de resina de Pínus e melhoramento 
genético. In: Shimizu JY. Pínus na silvicultura brasileira. Colombo: Embrapa. 49-74.

Shimoyama VRS, Wiecheteck MSS (1993). Características da madeira e da pasta termomecânica Pinus patula var. tecunumanii para produção de papel imprensa. Série Técnica IPEF, 6(27):63-80. ipef.br/publicacoes/stecnica/nr27/cap06.pdf

Sousa RC, Giovanini EP, Lima IL, Florsheim SMB, Garcia JN (2007). Efeito da idade e da posição radial na densidade básica e dimensões dos traqueídeos da madeira de Pinus taeda L. Revista do Instituto Florestal, 19(2):119-127. smastr16.blob.core.windows.net/iflorestal/ifref/RIF 19-2/RIF19-2_119-127.pdf

Torres LF, Melo R, Colodette JL (2005). Bleached kraft pulp production from Pinus tecunumanii (Eguiluz e Perry). Revista Árvore, 29(3):489-494. doi.org/10.1590/S0100-67622005000300017.

Trianoski R, Matos JLM, Iwakiri S, Prata JG (2014). Avaliação das propriedades mecânicas da madeira de especies de Pinus tropicais. Scientia Forestalis, 42(101):21-28.

ipef.br/publicacoes/scientia/leitura.asp?Article $=02$

$\&$ Number $=101$

Trianoski R, Matos JLM, Iwakiri S, Prata JG (2013). Variação longitudinal da densidade básica da madeira de espécies de Pinus tropicais. Floresta, 43(3):503-510. dx.doi.org/10.5380/rf.v43i3.28252.

Winck RA, Fassola HE, Área MC (2015). Efecto del raleo sobre las propiedades anatómicas de la madera de Pinus taeda. Maderas. Ciencia y tecnología, 17(2):391-406. dx.doi.org/10.4067/S0718$221 X 2015005000037$.

Zanchetta D, Macedo Reis C, Silva CEF, Silva DA, Luca EF, Fernandes FS, Lutgens HD, Tannus JLS, Pinheiro LS, Martins MRC, Sawaya R (2006). Plano de Manejo Integrado das Estações Ecológica e Experimental de Itirapina. São Paulo: Instituto Florestal. 318 p.

Zenid GJ (2007). Madeiras e suas características. In: Oliveira JTS, Fiedler NC, Nogueira M (ed). Tecnologías aplicadas ao setor madeireiro. Jerônimo Monteiro: Suprema Gráfica e Editora Ltda, p. $129-163$. 